\title{
Validation of Family Functioning Questionnaire (FFQ) with a Sample of Parents of Children with Autism Spectrum Disorder in Addis Ababa
}

\author{
Tadele Zebrea ${ }^{1}$, Belay Tefera ${ }^{2}$, Bhatara Mohit ${ }^{3}$, \\ ${ }^{1}$ Lecturer at Hawassa University and Doctoral Student at the School of Psychology, Addis Ababa University \\ ${ }^{2}$ Professor of Psychology, School of Psychology, Addis Ababa University \\ ${ }^{3}$ Consultant, United Arab Emirates
}

\begin{abstract}
Contextually validated measures of family functioning of parents with special needs are unavailable in Ethiopia. This study was aimed to validate the Amharic version of the Family Functioning Questionnaire (FFQ) with evidence from community samples of families of children with Autism Spectrum Disorder ( $n=72)$. Having translated the FFQ into Amharic, then the validation processes of assessing the psychometric properties (validity and reliability) of the instrument were conducted using experts' judgments, factor analysis, and internal consistency measures. The face and content validity assessments resulted in a measure that lends itself to the construct it intends to measure. The principal component factor analysis brought out two factors ( problem-solving and personal goal) with the majority of items demonstrated a factor loading above 0.5. The convergent validity of the FFQ was demonstrated via the Dyadic Adjustment Scale in which the observed significant intercorrelations suggested a strong construct validity of the Amharic FFQ. The internal consistency of the scale was also good, with a Cronbach's alpha of 0.82 for the whole scale and 0.91 and 0.82 for the problem-solving and personal goal sub-scales respectively. Hence, the Amharic version of the FFQ can be used for measuring the quality of family functioning in families of children with developmental disorders in the Ethiopian context.
\end{abstract}

Keywords: ASD, Family Functioning, personal goal, problem-solving, validation

\section{Introduction}

Family is the basic unit of society and is an important setting for individual physical and mental growth. A family affects the growth and development of every member and plays an important role in the normal operation process of the social system. Therefore, a family function is determined by the characteristics of the family itself and depends on social demand (Dia, \& Wang, 2015).

According to Beavers and Hampson (2000), family functioning could be conceptualized along two dimensions: family competence and family-style. Family competence refers to the family structure and capacity to adapt to changes through time. Family style is about the style and quality of family interaction. According to the model, a family within the harmonized structure with the capacity to adapt to changes and with stylistic quality interaction is regarded as a healthy functioning family.
Family functioning is the single most important determinant of the wellbeing and mental health of children and parents. A study by Keitner and Miller (1990) revealed that Poor family functioning was associated with more conflicts, less adaptability, and cohesion, as well as a disorganized pattern of everyday planning and assignment of chores. A crossectional study conducted on adults in China indicated that positive family functioning was associated with better mental status (Cheng, Zhang, Wang, Zhang, Ye, \& Liang, 2017). When the family functioning is compromised due to various social and personal factors, the whole family would be affected negatively as a unit. Particularly, parents are more vulnerable to the disturbance since they are the one who is in charge of the family. Their response and ability to manage the dynamics determine the effect on the family as a whole. It has been well-documented that parental mental health, which is often manifested by levels of anxiety, depression, and stress, is a positive predictor of the 
emotional and behavioral problems of children (Goelman, Zdaniuk, Boyce, Armstrong, \& Essex, 2014; Lereya \& Wolke, 2013).

The issue of family functioning is particularly crucial in families of children with disabilities in general. The birth of a child with disabilities brings unpredicted new demands and challenges to the parents (Dervishaliaj, 2013). Thus most parents of children with disabilities are faced with psychological problems such as stress, depression, and mental confusion (Bailey, Golden, Roberts, \& Ford, 2007; Mohan, \& Kulkarni, 2018), which is one of the major factors that impair the effectiveness of parenting (Guajardo, Snyder, \& Petersen, 2009). When the severity of the child's disability increases the demandingness of the case also increases (Hodapp, Glidden, \& Kaiser, 2005) that the effect gets prevalent on caregivers. Thus, raising, a child with severe developmental problems including Autism Spectrum Disorder observed to have pervasive effects on parents.

According to Reinchow, Steiner, and Volkmar (2012), Autism and the related pervasive developmental disorders are conditions demonstrated by delay and irregular development of social, communicative, and other important daily living skills, characterized by lack of social interest unusual sensitivity to the inanimate environment, difficulties with change, and idiosyncratic interests or preoccupations. The social and behavioral problems these children exhibit affect the family functioning to the extent that could jeopardize the whole family's wellbeing.

Family functioning has become an important area of concern in social science research as a determinant factor for family wellbeing, marriage stability, and marital adjustment. Exploring variables intended to inform the measurement of family functioning has been the interest of social science researchers to design appropriate tools that could be employed to assess the level of family functioning.

Several family functioning scales have been designed and being implemented in various community samples. For example, it is possible to mention the Family Environment Scale (Moos and Moos, 1981), Conflict Behavior Questionnaire (Prinz, Foster, Kent \& Leavy, 1979), Family Climate Scales (Schneewind, 1996), and Family Assessment Measure ( Skinner, Steinhauer \& Sitarenios, 2000) as some of the most commonly used measures of family functioning. However, the psychometric properties of many of the existing scales are determined based on evidence from samples of children, adolescents, and adults with typical development mainly from the west. Little effort has been done in designing family functioning measures particularly with families of children with disabilities and developmental disorders.

The levels and nature of functions of families of atypically developing children are different from families with typically developing children because atypical development demands many aspects of the family life (Compas, Jaser, Dunn,\& Rodriguez, 2012; Barros, César, Carandina, \& Torre, 2006; Miedema, Fortin, \& Easley, 2010). Particularly in contexts like this study area where there are limited opportunities of support and resources for parents, and highly characterized by the prevalent negative attitude towards children with disabilities and their parents, family functioning becomes worthy of study.

Much of the available family functioning measures validated on special populations intend to identify defects and malfunctioning in a family based on the assumption that children with disabilities disrupt family functioning and this would intern damage the family system. However, evidence suggests that having a child with developmental problems would not necessarily cause poor family functioning. Comparative studies reported mixed findings concerning the impact of rearing a child with chronic developmental problems on the family system. Some of these studies signified the negative impacts including poor marital satisfaction (Higgins, Bailey, \& Pearce, 2005; Gau, 2011), higher separation rate (Baeza-Velasco, Michelon, Rattaz, Pernon, \& Baghdadli, 2013; Hartley, Barker, Seltzer, Floyd, Greenberg, Orsmond, \& Bolt, 2010), and being more distressed (Brobst, Clopton, \& Hendrick, 2009; Dunn, Burbine, Bowers, \& Tantleff-Dunn, 2001). Others claim that raising a child with chronic problems enhances family harmony and connectedness by urging parents and siblings to increase time spent with one another and work together to cope with the situation and thereby make them to be more resilient to challenging circumstances (Bozo, Anahar, Ates, \& Etel, 2010). Still, other studies reported that there is no difference in the quality of family functioning between parents of children with disabilities and without disabilities (Hartley et al., 2010; Daire, Munion, \& Carlson, 2011).

Family functioning measures are unavailable in Ethiopia, particularly specific assessment tools that are intended to measure the quality of family functioning of 
families of children with developmental disorders. However, as stated by Roncone, Mazza, Ussorio, and police (2008) the measurement of family relationships in families of people with a mental problem has been one of the most interesting issues for the last 40 years among scholars in the west and yet much work remains to be done. As already mentioned the existing tools on family functioning are mainly validated on sample general population in a culture that has a different conception of having a child with a disability and are mainly intended to identify a problem in the family. The Family Functioning Questionnaire adapted and validated in this study is found to be better in focusing on children with disabilities and approaching the problem from the positivist perspective. Additionally, since the tool is at the preliminary stage of development it urges continuous validation works in different cultures.

Thus the main objective of this study was to adapt and validate a self-report instrument to ascertain the core aspects of family interpersonal functioning in the Ethiopian context particularly to be used for families of children with developmental disorders and chronic illnesses. More specifically, it attempts to determine the content validity, face validity, construct validity, and reliability of the Amharic version of the family functioning questionnaire.

The tool could be applied in identifying areas of psychoeducational intervention as well as progress and outcomes of interventions to inform and assist the efforts of family psychologists and practitioners. In addition to these, this validation study is conducted to adopt a tool of family functioning that could be used by researchers who have the interest to study family adjustment and functioning in the presence of children with developmental disorders.

\section{Methods and Materials}

In this methods section, participants of the study, instruments and variables, procedures followed, and data analysis techniques employed are presented and discussed in brief.

\section{Participants of the study}

The samples for this study were composed of 72 participants (parents of children with ASD), aged between 18 and 65 years, from Addis Ababa. The participants have enrolled their children either in Joy autism center or Nehemiya Autism center. All participants provided the following socio-demographic data: age, sex, educational status, marital status, occupation, and relationship with the child. For this validation study, being a main care provider for a child with ASD and being in a relationship (currently or at most before 6 months) were established as the inclusion criterion, and the following were established as exclusion criteria: (a) being a minor (less than 18 years old), (b) not being in a relationship at least for more than six months, and (c) not direct care provider for the child.

\section{Description of the Family Functioning Questionnaire}

The Family Functioning Questionnaire that was adopted and validated in this validation study was originally designed by Roncone, Mazza, Ussorio, police, Falloon, Morosini, and Cassacchaia and published in the Community Mental Health Journal in 2007. They intended to develop a self-completed measurement tool that could be used in the assessment of family functioning before and after a psycho-educational intervention.

The authors develop the first 60 item lists of questions on the three domains derived from the literature review and their personal experiences. After a series of trying out of the tool on the relatives and caregivers of schizophrenia patients at the psychiatry department of the University of L'Aquila, Italy, the researchers finally come up with a final English version tool with 24 items to be rated on a 4-point scale from 0 3 ("never", "sometimes", "often", "always"). In a general sense, high scores indicate greater family functioning, and low scores indicate poor family functioning.

The family functioning questionnaire was designed to involve three psychological constructs (problem-solving, communication, and personal goal) intend to measure the degree of problem-solving, personal goals, and communications in a family, and there were eight items for each sub-scale. Based on evidence from relatives of 92 caregivers of schizophrenic patients, the internal consistency of the scale in the original study was found to be satisfactory for the total scale $(\alpha=.84)$ as well as for sub-scales (problem-solving: 0.83 , communication: 0.71 , personal goal: 0.66) to justify their use. On the other hand, evidence from content, and construct validity indicated that the tool is a promising tool to measure family 
functioning in the presence of children with chronic problems.

\section{Procedures}

This assessment was performed from March to May 2019. To obtain an adequate sample of most representatives to the population, two autism centers were approached in Addis Ababa namely Nehemiya and Joy autism centers. These were the only two well-known centers providing services for children with an autism spectrum disorder in Addis Ababa. There were 68 and 56 children with ASD in Joy and Nehemiya autism centers respectively. During various times 56 parents from Joy center and 46 parents from Nehemiya (a total of 102 parents) were contacted at the center during consultation meetings and while they were bringing their children to the centers. From a total of 102 parents, 13 were disregarded because they were unable to meet the inclusion criterion and 89 were retained as samples for the study. Of 89 questionnaires distributed to the sample parents, 78 were returned ( $87.6 \%$ response rate). Finally, a total of 72 questionnaires were considered for analysis by rejecting 6 questionnaires due to their incomplete information.

It was the researcher who contacted the parents and gathered the data at the centers. Parents were informed about the objective of the assessment and their full consent was secured before administering the questionnaire. All of the participants who have taken part in the study were participated voluntarily and with full consent. The protocol was anonymous and contained simple introductory instructions that inform the purpose and instructions on how to provide the required information.

Rigorous content and face validity assessments were carried out before the tool was ready for administration. Then, based on the information gathered from the participants, validity, and reliability tests (Cronbach alpha) were computed by using relevant procedures that properly lend themselves to the required process.

Face validity to the tool was carried out to evaluate the appearance of the questionnaire in terms of feasibility, readability, consistency of style and formatting and the clarity of the language used. Here 4 experts (Ph.D. candidates of Addis Ababa University from the language and special needs department) were used to rate their agreement or disagreement on the specified issues and requested to indicate their comment on the questions that should be modified.

Content validity was undertaken to ascertain whether the content of the questionnaire was appropriate and relevant to the study purpose. According to Pilot and Hunger (1999), content validity is a vital procedure to ascertain the content reflects a complete range of the attributes under the study and is usually undertaken based on the judgment of seven and more experts. Indeed, 8 experts (psychologists who work as social workers in organizations that provide support to families of developmental disorder and who have expertise in the area) were used to judge the content validity of the questionnaire by comparing the constructs intended to measure against the specific question and were rated their judgments using 4 points Likert scale $(1=$ not relevant, $2=$ somewhat relevant, $3=$ relevant, $4=$ very relevant). According to Lynn (1996), items with an agreement rate of $7 / 8$ and above should be taken directly; $6 / 8$ should be modified and 5/8, and below should be rejected. Similarly, Lawshi's (1975) Content Validity Ratio (CVR) recommends retaining items with a CVR of 0.75 and above. These guidelines were used to revise and modify items upon the opinion of the experts.

As a measure of construct validity, factor analysis, and convergent validity were computed in addition to content validity. For convergent validity, the Dyadic Adjustment Scale (Spanier, 1976) was used. The Family Functioning questionnaire (FFQ) and Dyadic Adjustment Scale (DAS) were translated to Amharic and re-translated to English by 2 different language experts for language equivalence. The translated scales were administered to assess the level of family functioning and adjustment in the presence of children with ASD in the family. In FFQ, the participants were asked to rate the frequency of their experience for matters in the family related to problem-solving, communication, and personal goals. Similarly, DAS was also administered along FFQ to measure the degree of adjustment among couples under sub-scales of consensus (13 items), satisfaction (10 items), satisfaction (5 items), and affection (4 items).

\section{Data Analysis}

Data analysis was performed by employing appropriate procedures and techniques that helped to determine the psychometric property of the Family functioning Questionnaire. 
The face validity of the tool was evaluated based on experts' judgment. Here four evaluators assessed the consistency, format, and readability of each item of the measure. Similarly, the experts' judgment method was applied to assess the relevance of the content to the construct that the tool was supposed to measure. Eight experts were judged the relevance of each item for the construct that lends itself to measure and based on their opinion (Content Validity Ratio), appropriate modifications were effectuated on the items.

To establish the construct validity of the instrument, factor analysis, and convergent validity (correlation between FFQ and DAS) were utilized in addition to evidence from content validity. For construct validity, to statistically determine the items to the relevant construct, factor analysis was carried out. For factor analysis, the principal component analysis method was employed to decide the number of factors and to cluster items that have higher loading on them. To further evaluate the construct validity of the tool, the convergent validity test was computed by comparing the tool against the DAS measure. Pearson Correlation analysis was employed to find out the association of the whole items of the two measures as well as subscales.

The internal consistency of the measure was determined by analyzing Cronbach alpha. The reliability test was performed for the whole scale as well as for the subscales. Pearson correlation analysis was also computed to check the internal consistency among the subscales as well as the whole FFQ. All statistical analyses were done with SPSS for Windows version 25 .

The language adaption and cultural relevance issues were considered during the validation process through the involvement of language professionals' judgment on the tool. The appropriateness of the language, feasibility of the language to the audience, the relevance of the contents to the specific culture are evaluated and the necessary modifications were introduced on the tool based on the information gathered from experts.

\section{Results}

During the validation process, various activities had been carried out to attain an adapted tool that could effectively be applied for parents of children with developmental problems particularly children with Autism Spectrum Disorder. In this result section of the report, procedures followed and results attained are presented and explained under themes of the demographic characteristics of study participants, the face and content validity, construct validity, and internal consistency.

\section{Demographic Characteristics of the Study Participants}

In this validation study, a total of 72 mothers of children with Autism spectrum disorder have participated. Of the participants, $84.7 \%$ were married, $11.1 \%$ were divorced, and $4.2 \%$ were widowed. Among this study participants, $76.4 \%$ were women, and $23.6 \%$ were males. Concerning occupation, $50 \%$ were unemployed, $20.8 \%$ were government employees, and $29.2 \%$ were self-employed. In relation to the educational status of the participants, $12.5 \%$ were illiterate, $22 \%$ were at primary level education, $21 \%$ were at the high school level and $44 \%$ had a college diploma and above.

\section{Face and Content Validity}

The face validity of FFQ was evaluated based on the judgments of four experts and all four experts agreed that 20 items were essential and three of the evaluators forwarded their opinion on the need for improvement on the four items. The tool is revised and improved based on their opinion. This was conducted as a preliminary validation to make the tool ready for further inspection.

Eight experts comprised of individuals who have ample experience in working with families and children with disabilities were used to further evaluate the content validity of the FFQ. The Content Validity Ratio $\left(C V R=\frac{n e-\mathrm{N} / 2}{N / 2}\right.$, in which $\mathrm{n}_{\mathrm{e}}$ is the number of raters indicating "relevant" and $\mathrm{N}$ is the number of raters) was computed, and Lynn (1996) and Lawshi (1975) guidelines were used to select relevant items.

All the experts expressed their agreement on the relevance of the 19 items, and 7 experts were agreed on the relevance of the 3 items, and only 2 items were rated as relevant by 6 experts which later on modified by expressing it differently. Intensive revision and modification were carried out on the items based on the opinion of the experts to make FFQ suitable and appropriate to the constructs it intends to measure.

\section{Construct validity}

Factor analysis and convergent validity determination were carried out to examine the construct validity of the Family Functioning Questionnaire. 
Factor analysis: Factor analysis was computed on the 24 items of the family functioning questionnaire by subjecting it to the Principal Component Analysis (PCA). Before performing the PCA, the suitability of data for factor analysis was scrutinized. To verify that the data set is suitable for factor analysis, Pallant (2010) recommended that the Kaiser-MeyerOlkin Measure of Sampling Adequacy (KMO) value to be 0.6 or above and that Bartlett's Test of Sphericity value to be significant. Similarly, he recommends having many correlation coefficients in the correlation matrix with a value of 0.3 and above.

For the present study data set, the measure of sample adequacy value was 0.66 , exceeding the recommended value of 0.6 , and Bartlett's Test of Sphericity reached the level of statistical significance. Moreover, an inspection of the correlation matrix revealed the presence of many coefficients of 0.3 and above. Having fulfilled the above assumptions in supporting the factorability of the data, PCA was computed.

PCA revealed the presence of six components with Eigenvalues exceeding 1, explaining 31.8\%, $12.9 \%, 8.2 \%, 6.6 \%, 6.4 \%$, and $4.9 \%$ of the variance respectively in the first run. However, there were less than 3 items with high loading in the four factors extracted in the first run. An inspection of the screen plot also revealed a clear break after the second component. Based on evidence from several items with high loading in each factor and screen plot, it was decided to retain two components for further investigation. This decision was also supplemented by the results of Parallel Analysis, which showed only two components with Eigenvalues exceeding the corresponding criterion values for a randomly generated data matrix of 250 sample size ( 24 variables $\times 250$ respondents).

The analysis of the first run also indicated items with low loadings. Three items were eliminated ${ }^{1}$ from the factor pattern matrix of the FFQ when the factor loading was less than 0.5 . The elimination of such items was confirmed by Steven's (2002) guideline statistical significance for interpreting factor loading. Steven's Guideline is based on sample size and suggests that the statistically acceptable loading for 50 participants is 0.72 , for 100 participants 0.51 , and for 200-300 participants 0.29-0.38. The sample size used in the FFQ validation process was 72 : as a result, three items with a loading $<0.5$ were deleted. The 18 items with loading $\geq$ 0.5 were accepted. Three remaining items had a loading of $<0.5$ but were accepted because it was important to the relevant factor. The final PCA of the two-factor solution with 21 items accounted for $45.8 \%$ of the total variance with Component 1 contributing $32.27 \%$ and Component 2 contributing $13.53 \%$. To aid in the interpretation of these two components, oblimin rotation was performed. The rotated solution revealed the presence of a simple structure with both components showing many strong loadings. The factor loading of the final PCA and their factorial weightings are shown in Table 1.

Table 1:

The results of the final two-factor solution of the FFQ according to the Principal Component Analysis with direct Oblimin rotation and the internal consistency of each factor

Items
Problem Solving ( $a=0.91)$
When I say to a family member that they have done something that I do not approve of, I politely tell them
to offend them personally.
When a decision has been made about what to do about a family problem, we all lend a hand to carry it out.
If we have a family problem we all meet together to discuss it.

\footnotetext{
${ }^{1}$ Deleted Items

7. When we have dealt with a problem in the family we usually discuss what we have done and whether it helped.
}

21. If one of my family members does something kind for me I almost never thank then because I do not think it is really necessary.

24. I can easily find interests that keep me busy. 
If I am unhappy with the behavior of someone in my family I tell them and suggest some ways that we can get along better.

If someone in the family does something kind for me, I thank them.

When someone in my family does or says something I like, I tell them openly that I am pleased.

I find it helpful to tell what I think about a problem in my family because they seem to take account of my opinion.

When we have a problem in the family I can suggest solutions without worrying about being criticized.

When I need my family to help me with something I ask them kindly and do not make demands or orders.

In our home, it is difficult to decide how to solve a problem because we never agree about anything.

When I am angry with someone in the family I tend not to speak to him/her.

I can talk with my family about the things that make me unhappy.

When we have a problem in our family we plan together what to do about it.

In the family, we collaborate to find the best way to solve our problems.

Personal Goal ( $a=0.82)$

I manage to do some things alone, without my family.

It is important that everyone in the family has time for themselves.

Despite many problems I have to deal to deal with in my family, I always find little time for myself.

I have to deal with so many difficulties in my family that I have almost completely given up on my interests.

I succeed in doing what I promise myself to do.

I have little time for my hobbies and interests because I spend most of my free time caring for my family.

I meet friends outside my family.

Convergent validity: the Dyadic Adjustment Scale (DAS) was used to compute the convergent validity of FFQ with the assumption that since the DAS scale is a well-established tool to measure family

Table 2:

\section{Correlation between the FFQ and DAS subscales}

\begin{tabular}{lllll}
\hline & & & Family & Functioning \\
& Problem-solving & Personal Goal & Total & \\
\hline Dyadic Consensus & $.283^{*}$ & $.330^{* *}$ & $.341^{* *}$ & \\
Dyadic satisfaction & .167 & .089 & .175 & \\
Dyadic cohesion & $.266^{*}$ & .111 & $.272^{*}$ & \\
Affective expression & $.331^{* *}$ & .117 & $.334^{* *}$ & \\
Dyadic total score & $.352^{* *}$ & $.298^{*}$ & $.396^{* *}$ & \\
\hline
\end{tabular}

$* \mathrm{p}<0.05, * * \mathrm{p}<0.01$

Table 2 above indicates the correlation coefficients between the FF subscales as well as total scores and DAS subscales and the total scores. As expected, positive and statistically significant correlations were demonstrated among the Problem Solving subscale of FF and Dyadic Consensus $(r=0.28$, $p<0.05)$, dyadic cohesion $(r=0.27, p<0.05)$, and affective expression $(r=0.33, p<0.01)$ subscales of DAS. The problem-solving subscale also demonstrated a statistically significant positive relationship with DAS total score $(r=0.35, p<0.01)$. The FF Personal Goals relationships, demonstrating a high correlation with this scale is an important input for FFQ that it fits for the purpose. 


\section{Reliability}

The internal consistency of FFQ was analyzed by computing Crombach alpha and was found to be 0.82 , which indicates a high correlation between the items and the questionnaire is consistently reliable. The Crombach alpha was also computed for each of the two subscales and it was found to be 0.91 and 0.82 for problem-solving and personal goal subscales respectively. Based on
Kline's criterion (2000), reliability coefficients of more than 0.7 confirm internal consistency. Therefore, the results indicate that FFQ passes Kline's criterion for internal consistency.

Similarly to evaluate the internal consistency among subscales as well as the total scale of FFQ, Pearson correlation analysis was computed.

Table 3:

Correlation between the FF subscales

\begin{tabular}{llll}
\hline & $\begin{array}{l}\text { Problem- } \\
\text { solving }\end{array}$ & $\begin{array}{l}\text { Personal } \\
\text { Goal }\end{array}$ & $\begin{array}{l}\text { Family } \\
\text { total }\end{array}$ \\
\hline $\begin{array}{l}\text { Problem Solving } \\
\text { Personal Goal }\end{array}$ & $\begin{array}{l}.253^{*} \\
.972^{* *}\end{array}$ & $.428^{* *}$ & - \\
Family functioning total & &
\end{tabular}

As stated in Table 3 above, the correlation between the FFQ subscales as well as the total score fluctuated from moderate to high, which indicates that the constructs are related to one another. The correlation coefficient between personal goal and problem solving was found to be positive and statistically significant $(r=0.253, p<0.05)$. Similarly, the correlation coefficient was statistically significant and positive between the total FF score and problem-solving $(r=0.92, p<0.01)$ and personal Goal $(r=0.428, p<0.05)$.

To sum up, as indicated in table 1 , the final family functioning questionnaire consists of 21 items within two subscales. Subscale one, "problem-solving", accounted for $32.27 \%$ of the total variance. This factor comprises fourteen items and reflects information about how the family reacts to a problem individually or as a group. The loading of items in this subscale ranges from 0.762 to 0.519 . Subscale 2: "personal goal" accounted for $13.53 \%$ of the variance and includes seven items with factor loadings ranging from 0.728 to 0.371 . These items measure people's opinions about important personal goals in their life.

\section{Discussion}

The main purpose of this study was to examine the psychometric properties of the Family Functioning Questionnaire based on scores obtained from a community sample of families of children with Autism Spectrum Disorder. The results depict that the FFQ is a valid tool to measure the quality of relationships in a family. The confirmation of the evaluators along with the content validity ratio determined in the study supplemented the finding. This finding is in line with Lynn's (1996) and Lawishi's (1997) guidelines and recommendations proposed for content validity determination.

The result of this validation study is also indicated that the structure of the present scale is simple and consists of two factors, which is somehow similar to that proposed by the author of the initial study (Roncone, et al., 2007). In the original tool, the authors identified three psychological constructs (problem-solving, communication, and personal goal), however, in this study, the factors are reduced to two (problem-solving and personal goals). This may be attributed to the fact that effective communication is one of the components of problem-solving in a family. These results also coincide somehow with evidence found in various other studies (Byles, Byren, Boyle \& Offord, 1988; Byren, Offord \& Boyle, 1992; Fisher 1976; Kim \& Kim, 2007). Similarly, evidence of convergent validity of the Family Functioning Questionnaire was demonstrated by a pattern of correlations with the relevant measures of marital satisfaction, the Dyadic Adjustment Scale, that was in line with the theoretical predictions. This finding is consistent with the previous studies that reported family functioning measures are significantly related to measures of marital satisfaction and relationship quality (Funk, \& Rogge, 2007; Heyman, Sayers, \& Bellack, 1994). 
This simple factor model leads to a single global score for family functioning, which facilitates the practical use of this instrument for evaluating and diagnosing the functional problem in community populations particularly in families of children with developmental disabilities.

Concerning the internal consistency of the FFQ, the tool demonstrated high internal consistency $(\alpha=0.82)$. Evidence of internal consistency of problemsolving and personal goal subscales was also demonstrated acceptable reliability. All inter-item correlations were also within an acceptable range, indicating good internal consistency. These findings are in line with the previous studies which reported that measures of family functioning and dyadic relationship demonstrate high internal consistency (Roncone, et al., 2007; Doane, Falloon, Goldstein, \& Mintz, 1985).

In summary, the FFQ is a reliable and valid tool for measuring the overall quality of family functioning and relationship in families of children with developmental disabilities. This inventory is short and easy to use the tool and can be administered in both research and clinical applications.

\section{Conclusions}

In this validation study, rigorous scientific procedures were followed to adapt and validate the Family Functioning Questionnaire that could be used in the Ethiopian context. The findings revealed that the adopted tool is valid and reliable particularly in measuring family functioning in the presence of children with developmental problems. However, several limitations should be considered when interpreting the results of this study. First, although the criteria of sample adequacy were achieved during the analysis process, this study is not conducted on a large sample size. In addition to that, the samples were not clinical samples that were experiencing a real problem due to the condition of their children. Future research should analyze whether or not the results are maintained for larger problematic families who need therapeutic help. Finally, although the confidentiality of the data has been assured, no social desirability scale was administered. This factor may distort the responses provided by the participants.

\section{Recommendations}

Families of children with developmental problems in Ethiopia are the most neglected ones. The existing support from various organizations focuses on the provision of assistive devices and materials for children. To support parents and enhance the resilience of a family in demanding situations, extensive studies should be conducted in determining measurement tools that could help to effectively identify intervention areas. This issue is particularly important in the Ethiopian context that using tools developed elsewhere in normative among researchers. There are unique cultural, psychological, and social elements that demand the development of measurement tools that could help to effectively trace areas of intervention in the community in general.

\section{References}

Barros, M. A., César, C. G., Carandina, L., \& Torres, G. D. (2006). Social Inequalities in the Prevalence of Chronic Diseases in Brazil. Ciência \& Saúde Coletiva, 11, 911-926.

Bailey, J.R.D.B., Golden, R.N., Roberts, J., Ford, A. 2007. Maternal depression and developmental disability: Research critique. Journal of the Mental Retardation and Developmental Disabilities Research Reviews, 13(4), 321-329

Beavers, W. R., \& Hampson, R. B. (2000). The Beavers systems model of family functioning. Journal of Family Therapy, 22(2), 128-143.

Bozo, O., Anahar, S., Ates, G., \& Etel, E. (2010). Effects of illness representation perceived quality of the information provided by the health-care professional and perceived social support on depressive symptoms of the caregivers of children with leukemia. Journal of Clinical Psychology in Medical Settings, 17(1), 23-30.

Brobst, J. B., Clopton, J. R., \& Hendrick, S. S. (2009). Parenting Children with Autism Spectrum Disorders: The Couple's Relationship. Focus on Autism and Other Developmental Disabilities, 24 , http://dx.doi.org/10.1177/1088357608323699

Byles, J., Byrne, C., Boyle, M.H., Offord, D.R. (1988). Ontario Child Health Study: Reliability and Validity of the General Functioning Subscale of the McMaster Family Assessment Device. Family Process, 27: 97-104.

Byrne, C.M., Offord, D.R., \& Boyle, M.H. (1992). Family Functioning and Emotional/Behavioral Disorders in Children: Results from the Ontario 
Child Health Study. Ministry of Community and Social Services, Ottawa.

Cheng, Y., Zhang, L., Wang, F., Zhang, P., Ye, B., \& Liang, Y. (2017). The effects of family structure and function on mental health during China's transition: a cross-sectional analysis. BMC Fam Pract 18, 59. https://doi.org/10.1186/s12875017-0630-4

Compas, B. E., Jaser, S. S., Dunn, M. J., \& Rodriguez, E. M. (2012). Coping with Chronic Illness in Childhood and Adolescence. Annu. Rev. Clin. Psychol. 8:455-80. Retrieved from http://clinpsy.annualreviews.org

Dai, L.T. \& Wang, L.N. (2015) Review of Family Functioning. Open Journal of Social Sciences, 3 ,

134-141.

http://dx.doi.org/10.4236/jss.2015.312014

Daire, A., Munyon, M., Carlson, R., Kimemia, M., \& Mitcham, M. (2011). Examining Distress of Parents of Children With and Without Special Needs. Journal Of Mental Health Counseling, $33(2)$, 177-188. https://doi.org/10.17744/mehc.33.2.qu73p0317 $6337 \times x 1$

Dervishaliaj, E. (2013). Parental Stress in Families of Children with Disabilities: A Literature Review. Journal of Educational and Social Research; 3(7), 579-584. Doi:10.5901/jesr.2013.v3n7p579.

Doane, J. A., Falloon, I. R., Goldstein, M. J., \& Mintz, J. (1985). Parental Affective Style and the Treatment of Schizophrenia: Predicting Course of Illness and Social Functioning. Archives of General Psychiatry, 42, 34-42.

Dunn, M.D., Burbine, T., Bowers, C.A., \& TantleffDunn, S. (2001). Moderators of stress in parents of children with autism. Community Mental Health Journal. 37:39-52.

Fisher, L. (1976). The dimension of Family Assessment: a Critical Review. Journal of Marriage and Family Counseling, 367-382.

Funk, J.L., \& Rogge, R.D.(2007). Testing the ruler with item response theory: Increasing precision of measurement for relationship satisfaction with the Couples Satisfaction Index. $J$ Fam Psychol,21(4),572.

Gau, J. M. (2011). The convergent and discriminant validity of procedural justice and police legitimacy: An empirical test of core theoretical propositions. Journal of Criminal Justice, 39(6),
489-498.

https://doi.org/10.1016/j.jcrimjus.2011.09.004

Goelman, H., Zdaniuk, B., Boyce, W. T., Armstrong, J. M., \& Essex, M. J. (2014). Maternal mental health, child care quality, and children's behavior. Journal of Applied Developmental Psychology, 35, 347-356. http://doi.org/3qz

Hartley, S.L., Barker, E.T., Seltzer, M.M., Floyd, F.J., Greenberg, J.S., Orsmond, G.I., \& Bolt, D. (2010). The relative risk and timing of divorce in families of children with an autism spectrum disorder. Journal of Family Psychology. 24:440-457.

Heyman, R.E., Sayers, S.L., Bellack, A.S.(1994). Global marital satisfaction versus marital adjustment: an empirical comparison of three measures. $J$ Fam Psychol, 8(4), 432.

Higgins, D., Bailey, S., \& Pearce, J. (2005). Factors associated with functioning style and coping strategies of families with a child with an autism spectrum disorder. Autism, 9(2),125-137. doi.org/10.1177/1362361305051403

Hodapp, R.M., Glidden, L.M., \& Kaiser, A.P. (2005). Siblings of persons with disabilities: toward a research agenda. Mental Retardation, 43 (5), 334338.

Kim, H.S., \& Kim, H.S. (2007). Development of a Family Dynamic Environment Scale for Korean Adolescents, Public Health Nursing, 24(4): 372-381.

Kline P. (2000). A Psychometrics Primer. Free Association Books: London.

Kogan, J.N., Edelstein, B.A., McKee, D.R. (2000). Assessment of anxiety in older adults: current status. J Anxiety Disorder 14, 109-132.

Lereya, S. T., \& Wolke, D. (2013). Prenatal family adversity and maternal mental health and vulnerability to peer victimization at school. Journal of Child Psychology and Psychiatry, 54, 644-652. http://doi.org/3q6

Lynn, M.R. (1996). Determination and quantification of content validity. Nursing Research, 35, 382385.

Miedema, B., Fortin, F., \& Easley, K.J. (2010). "You can only take so much and you took everything out of me": Coping strategies used by parents of children with cancer. Palliative and Supportive Care, 8 , 197-206.

DOI:10.1017/S1478951510000015 
Mohan, R. \& Kulkarni, M. (2018). Resilience in parents of children with intellectual disabilities. Psychology and Developing Societies, 30(1), 1943.

Moos, R. H., \& Moos, S. (1981). Family environment scale manual. Palo Alto, California: Consulting Psychologists Press.

Pallant, J. (2010). SPSS Survival Manual: A step by step guide to data analysis using S PSS $\left(4^{\text {th }} \mathrm{ed}\right)$. Allen \& Unwin Book Publishers, Australia

Pilot, D., \& Hunger, B. (1999). Nursing research: principals and methods. Lippincott Williams \& Wilkins: Philadelphia.

Reichow, B., Steiner, A.M., \& Volkmar, F. (2012). Social skills groups for people aged 6 to 21 with autism spectrum disorders (ASD). Campbell Systematic Reviews, DOI: 10.4073/csr.2012.16

Roncone, R., Mazza, M., Ussorio, D. \& police, R. (2008). The Questionnaire of Family Functioning: A Preliminary Validation of a Standardized Instrument to Evaluate Psychoeducational Family Treatments. Community Mental Health Journal, 43, (6), DOI: 10.1007/s10597-007-9093-8

Skinner, H., Steinhauer, P. \& Sitarenios, G. (2000). Family Assessment Measure and Process Model of family functioning, Journal of Family Therapy, 22: 190-210.

Spanier, G.B. (1976). Measuring Dyadic Adjustment: New Scales for Assessing the Quality of Marriage and Similar Dyads. Journal of Marriage and the Family, 38, 15-28.

Stevens, J. (2002). Applied Multivariate Statistics for the Social Sciences (4th Edition). Mahwah, NJ: Lawrence Erlbaum Associates. 